\title{
LAS VICISITUDES DE LA NEGACIÓN
}

\section{Mercédesz Kutasy}

Eötvös Loránd Tudományegyetem, Budapest

mkutasy@gmail.com

Resumen: El presente texto, partiendo de las reflexiones de David Antin, busca precisar y a su vez ampliar los marcos del cuento. Se observarán las diferentes definiciones y usos del género según épocas históricas y periodos artísticos, y se intentará detectar las diferencias sustanciales entre cuento, cuento experimental y anticuento comparando antologías y fragmentos de textos.

Palabras clave: cuento, cuento experimental, anticuento, género

\section{VICISSITUDES OF NEGATION}

Abstract: This paper pretends, starting at David Antin's considerations, specify, and at the same time extend the frames of the short story. We will examine the different definitions and purposes of the genre according to historical and artistic periods, as we will attempt to detect essential differences between short story, experimental short story, and anti-story, basically comparing anthologies and textual fragments.

Keywords: short story, experimental short story, anti-story, genre

DOI: $\underline{\text { https://doi.org/10.24029/lejana.2019.12.393 }}$

Recibido: el 15 de junio de 2019

Aceptado: el 30 de agosto de 2019

Publicado: el 30 de octubre de 2019 
David Antin, en su texto titulado "The Stranger at the Door", reflexiona acerca de un texto del escultor George Brecht. El texto, publicado en la revista some/thing por el autor del artículo y Jerome Rothenberg, lleva el encabezamiento "Excerpts from / Gloss For An Unknown Language" e incluye líneas (no sucesivamente) numeradas, en las que se describen caracteres de una lengua desconocida y a veces también se añaden alusiones a la opacidad del significado de los mismos. Tras la cita, Antin explica que el texto de George Brecht originalmente formaba parte de una serie impresa sobre cartulinas individuales y fue publicada por George Maciunas bajo el título WaterYam, en cajas de cartón de su propio diseño. El artículo de Antin sigue así:

En la revista aparecieron bajo el título

GEORGE BRECHT

Dances, Events \& Other Poems

entre el índice y un capítulo al que las notas sobre los autores refieren como aquel que contiene "la novela enciclopédica en proceso" de Rochelle Owens, titulada Elga's Incantation. Si mal no recuerdo, el título viene de mi coeditor, ya que Brecht, en ese momento en el extranjero, no nos dejó más que una pila de copias de manuscritos. Por motivos de economía y claridad pusimos dos o tres de las piezas más cortas en la misma página, pero con las márgenes desajustadas para acentuar que se trataba de unidades independientes. La tipografía y el título sugieren inequívocamente que los textos han de ser considerados poemas. ${ }^{1}$ (Antin, en línea)

De esta breve cita se vislumbra que la elección de un género es, para no decir otra cosa, sumamente accidental. La obra, dice Antin, consiste de una lista de signos invisibles para el lector, pero descritos, aunque, en algunos casos, de significado no evidente. El título precisa que los textos forman parte de una serie titulada Danzas, Eventos y Otros Poemas, aunque no se llega a saber cuáles de los textos pertenecerían en qué género - aun así el final del título (y Otros Poemas) nos hace intuir que las danzas y los eventos también son, al mismo tiempo, poemas- Todos estos textos se recopilan a base de una serie de cartulinas hechas por el autor pero publicadas en una caja, en el marco del Fluxus, por George Maciunas. Y en la revista hasta la forma original se pierde y se altera, porque Brecht está en el extranjero "y no nos dejó más que una pila de copias de manuscritos", y también "por motivos de economía y claridad". El significado se esconde tras una "lengua desconocida", la forma se altera constantemente y el género se nos escapa de las manos. El título hasta nos empuja fuera del ámbito de lo literario denominando las pocas líneas de la obra como "danzas" y "eventos", a la vez igualándolos con el término "poemas". Y la confusión aumenta ya que los creadores no añaden ninguna explicación que precisara cómo puede interpretarse un texto como danza, a su vez cómo se convierte esta danza en poema, etc. Surge entonces la duda: si es tan difícil afirmar cualquier cosa sólida e inmutable sobre una obra de arte en particular, ¿para qué insistir en los géneros que al parecer complican aun más la

\footnotetext{
1 "In the magazine they appeared under the title / GEORGE BRECHT / Dances, Events \&Other Poems /between the Table of Contents and a chapter of what the Contributor's Notes referred to as Rochelle Owens' "encyclopedic novel-in-progress," Elga's Incantation. The title was as I remember supplied by my co-editor, because Brecht was out of the country and had simply left us a pile of manuscript copy. Together with the layout, in which the smaller pieces, for purposes of economy and clarity, were printed two or three to a page with their margins staggered to maintain their separate identities, the title tended to suggest somewhat equivocally that these texts were all to be considered poems." La traducción al castellano es mía.
} 
interpretación? Sin la meticulosidad teórica de precisar el género de estas líneas, nos hallaríamos en el feliz terreno de la recepción subjetiva y — para qué disimular — aun así sería bien complicado, si no imposible entenderlas. Pero así es, sin duda, peor.

El cuento es uno de los géneros cuya teoría ha llenado bibliotecas y anaqueles, sin embargo, hemos de reconocer la imposibilidad de sumar todas aquellas características narrativas (por cierto, después del ejemplo anterior, ¿tienen que ser necesariamente narrativas?) que lo determinen y definan con exactitud, pero sin caer en lo superficial. Anderson Imbert en su Teoría y técnica del cuento esboza la cronología y cambio de significado de la palabra y cita a Croce, quien afirma que los géneros responden a la necesidad histórica de organizar el caos entendido como el conjunto de todas las obras literarias escritas hasta el momento. El autor también subraya que el uso de la palabra cuento varía según la época: en la Edad Media todavía se vincula con el concepto de contar (números), el ejemplo citado es Disciplina clericalis de Pedro Alfonso (ca. 1062), narración en el que se cuentan las ovejas de un aldeano hasta dos mil, para que un rey logre dormir; en el Renacimiento la palabra se enriquece con el criterio de la oralidad y es utilizada en contraste con la novela, de carácter eminentemente escrito.

El cuento muchas veces aparece en comparación: suele ser más corto que la novela, más largo que el minicuento (y ¿cómo distinguir entonces minicuento y, por ejemplo, tuiteratura?); Anderson Imbert llama sin embargo la atención en que no necesariamente ha sido así en todas las épocas: sigue sus reflexiones sobre el Renacimiento y menciona que para Cervantes " $[1]$ a diferencia entre novela y cuento no era [...] cosa de dimensiones en el espacio sino de actitud: espontánea en el cuento, empinada en la novela. Novelar es inventar; contar es transmitir una materia narrativa común" (2007: 17). Con el Romanticismo la noción llega a incluir el verso, eso sí, siempre y cuando este sea "de carácter fantástico", y para el siglo XIX ya se emplea "para narraciones de todo tipo" (17).

Si observamos el fenómeno desde el punto de vista práctico, en vez de el de la teoría, en las antologías de cuentos hallamos en su mayoría textos con pocos personajes (¿podremos precisar cuántos?), un hilo narrativo, en un principio también nos sirven las pautas establecidas por Horacio Quiroga; y sin embargo siempre hay alguna que otra exepción. El cuento borgiano es muchas veces más bien ensayo, hay cuentos que compiten en su extensión con la novela corta y tan solo depende de la decisión del editor - y por supuesto de la del tipógrafo- hacia dónde se inclina nuestra consideración. En los dos volúmenes de la antología El cuento hispanoamericano en el siglo XX editado por Fernando Burgos, el texto más corto apenas ocupa tres páginas, el más largo casi llega a treinta, y entre los textos clásicos encontramos monólogos interiores sin puntuación de una sola frase (por ejemplo, "Las cosas andan mal, Carmelo Rosa" del peruano Julio Ramón Ribeyro), pero también textos más tradicionales de Julio Cortázar o de Carlos Fuentes. El texto de Ribeyro bien podría formar parte de otra antología, de cuento experimental, como la editada por László Scholz, y de hecho en el tomo Prosas de mareo encontramos un texto de Rosario Ferré ("Carta") que comparte gran cantidad de las características del de Ribeyro. La extensión en ambos casos es igual, tres páginas; ambos son monólogos interiores, en ambos casos falta la puntuación excepto de un único punto final; eso sí, en "Carta” de Ferré aparecen párrafos, elemento estructurador que falta del texto de Ribeyro y el cuento, así como cada uno de sus párrafos empieza con minúsculas. Aun así parece una diferencia estructural bien 
insignificante como para explicar por qué uno de los textos figura en una antología acompañado de otros textos más bien clásicos, y el otro por qué es ejemplo de la experimentación. Hallar la respuesta no es meta de este ensayo, el ejemplo sirve nada más para ilustrar la amplitud de los marcos de cada género. El cuento experimental (muchas veces de extensión más corta que el cuento tradicional) se empeña en contradecir alguna de las características del cuento: en Prosas de mareo se hallan textos que juegan con la tipografía ("Muerto - Martín Valdez - muerto" de Víctor Retamoza, "Nínive" de Héctor Libertella), otros que fragmentan el cuerpo del texto en unidades numeradas (como es el caso de "Mnemothreptos" de Salvador Elizondo), privan el texto de la presencia de personajes para ofrecer una generalización al máximo de los elementos narratológicos ("Genealogía" de Felisberto Hernández), hacen uso de formas ajenas ("Diario" de Felisberto Hernández o "Solicitud" de Mariluz Rodríguez Castañeda), mezclan la corta extensión del cuento con técnicas o denominaciones teatrales ("Comedia inmortal" de Pablo Palacio o "Algo en la oscuridad" de José Emilio Pacheco). Enrique Anderson Imbert a su vez menciona una antología anterior, la de Philip Stevick: AntiStory: an Anthology of Experimental Fiction, ${ }^{2}$ libro cuyo título sugiere que para el editor "anticuento" y "cuento experimental" podrían ser sinónimos. En este tomo vemos una estructuración de los textos "según la clase de agresión que se cometa contra el clásico arte de contar: «contra la imitación de la vida», «contra la realidad», «contra el acontecer de hechos», «contra el tema», «contra las experiencias normales», «contra el análisis», «contra el significado», «contra la proporción en el espacio» [...], contra contra contra... El anticuento sería, pues, algo así como un subgénero reaccionario" (Anderson Imbert, 2007: 19) Tras observar el contenido y las categorías (etiquetadas o no) de las dos antologías, queda claro la similitud entre las posibilidades de la desviación de la norma del "cuento experimental" y del "anticuento".

En algunos casos el texto aparentemente contradice a su propio género, como es el caso de "Etc.” de Luis Rafael Sánchez (Scholz, 2001: 189-193), que empieza así: “Aquí va un cuento que no es un cuento". En la segunda parte de la frase el narrador manda a su lector al territorio de la realidad en vez de la ficción, cuando dice: "porque ocurrió ayer mismo en la esquina de la diecisiete". El carácter oral del fragmento lleva lejos de lo que en la actualidad llamaríamos "cuento" para acercarnos a la acepción renacentista de la palabra, vinculada a la génesis espontánea. En este caso el lector se coloca en la postura del transeúnte que se entera de un fragmento de vida sin querer. Y sin embargo el texto de Sánchez empieza diciendo "aquí va un cuento" y la negación subsiguiente parece no negar, más bien matizar esta primera afirmación. El "cuento que no es un cuento" en este contexto apuntaría más bien a la experimentación y sería "un cuento que no lo es en el sentido clásico de la palabra" y no "un cuento que se autoexcluye del género". Para aquello, por definición, tendríamos otra palabra, el anticuento.

En un principio, el prefijo anti sugiere una condición opuesta al del cuento, por lo tanto como lector espero algo que niegue alguna característica sustancial del género; una reacción entonces, el contra de Stevick. Pero ¿hasta qué punto será posible la negación? Cuando defino el cuento como un "género corto", el anticuento tendría que ser largo o al contrario, tan corto que se autoexcluya por el otro extremo. Si el cuento es "un género

\footnotetext{
${ }^{2}$ Nueva York: The Free Press, 1971.
} 
prosaico corto", el anticuento sería tal vez un género poético; si el cuento es un texto, el anticuento debería de ser algo bien distinto; y si considero el marco de la literatura como referente imprescindible del cuento, el anticuento quedaría fuera de ella. Y aun así es más que problemático determinar si algo pertenece o no en una categoría determinada. Como dice Antin:

La pregunta clave respecto a los géneros indaga acerca de cuáles son las condiciones necesarias y suficientes para poder incluir una obra en algún género [...]. A la vez cada vez más obras pretenden ser incluidas en los géneros ya establecidos y es preciso decidir sobre su destino. La historia del arte moderno abunda en ejemplos cuando algún crítico conocido afirma que una obra no es "pintura", "música", "teatro" o "baile", a su vez otros tienen argumentos de sobra para calificar la obra en cuestión como "pintura", "música", "teatro" o "baile". (en línea)

A continuación Antin afirma que la inclusión o, al contrario, exclusión de una obra en o de un género tiene que ver siempre con algún "atributo indispensable" que la hace partícipe o ajena al género en cuestión. Sin embargo, sigue Antin, “este atributo indispensable, según nos enteramos casi siempre, caracteriza tan solo un subgénero favorecido". Hemos visto antes: si considero como "atributo indispensable" de un cuento la extensión, la novela corta ya queda fuera, y como tal, en comparación, es anticuento. Si me conformo con que el cuento es un género literario hecho con fines estéticos, tengo que encontrar algo ajeno a la literatura, un objeto totalmente cotidiano que no tenga nada que ver con lo estético. Un objeto de uso práctico, elegido imparcialmente: por ejemplo, una silla, casi un readymade, el anticuento por excelencia.

Ahora, veamos el fenómeno del revés: si nuestra silla utilizada como ilustración de la plasticidad de la definición es el anticuento por excelencia, el ejemplo tendría que funcionar también a la inversa. La hipotética silla se clasifica dentro del género anticuento. Pero, pensándolo en detalle, bien podría ser antipoesía o antidanza, contrario a cualquier otra cosa del que no forma parte. Sin embargo, surge otra duda acerca de la denominación: así como anti puede significar absolutamente contrario, a veces también se usa en el sentido de "contrario, conservando sus elementos constitutivos". Me refiero a expresiones tipo antítesis, que contradicen a la tesis siguiendo cada uno de sus elementos constitutivos. La antítesis no es un sistema arbitrario que no tiene nada que ver con la tesis; al contrario, sigue sistemáticamente la lógica de la primera para refutarla, es decir, en su estructura, razonamiento o tema se hallan y se reconocen las huellas de la tesis.

Si la definición en sí no nos sirve de orientación, vayamos de lo particular a lo general y observemos algunos ejemplos de aquel corpus que se autodefine como anticuento, a ver si con este método se logra destilar la definición.

La antología de Stevick ya la hemos mencionado, y el título de este tomo en sí bastaría para minar la más mínima posibilidad para poner orden en el "caos" mencionado por Croce. En 2017 se publicó el tomo de Óscar Rodrigo titulado Anticuento, historia cuyos personajes

\footnotetext{
3 "These questions of necessary and sufficient conditions qualifying a work for entry in a genre is really a central issue for the concept of genre [...]. But new works are continually being proposed for inclusion in established genres and judgments are constantly being made about the suitability of their candidacy. The history of modern art is filled with accounts of well known critics confronting works that they declare are not 'painting' or 'music' or 'theater' or 'dance', only to be answered by others that what they have been confronting is indeed and for certain very good reasons 'painting', 'music', 'theater' or 'dance'." La traducción es mía.
} 
proceden de los cuentos tradicionales, sin embargo la trama se desarrolla en el siglo XXI. Lo que al género se refiere, este se define como "una novela de género fantástico con altas dosis de humor y aventura". ${ }^{4}$ Anticuento sería entonces una novela con personajes prestados de los cuentos infantiles cuyo elemento estilístico más llamativo es el humor y la ironía. Ahora, Don Quijote, que trabaja igualmente con muchos intertextos, altas dosis de humor y aventura, ¿será también anticuento?

En Anticuentos del mexicano Antonio Domínguez Hidalgo igualmente encontramos intertextos de cuentos infantiles, esta vez de las versiones hollywoodenses de Walt Disney. El tomo otra vez promete irreverencia, chiste y hasta carcajada cuyo ritmo acompañaría los versos de Domínguez Hidalgo. La transcripción y burla de los textos clásicos y la alteración de la forma original (en vez de prosa de corta extensión, novela o poesía) parecen constantes del género.

Anticuentos de Juan José Millás sin embargo se introduce como "una serie de artículos" anteriormente publicados por la prensa que "por sus características están más cerca de los textos de ficción, de la fábula o del microrrelato fantástico". ${ }^{5}$ La extensión, en este caso, no dista de la del cuento tradicional, y el texto está escrito en prosa: la diferencia, entonces, residiría en el carácter de los textos, que en vez de narrar historias, meditan acerca de la naturaleza de ciertos fenómenos. Lejos de ser una contradicción total del cuento, el método evoca inmediatamente textos como aquellos de Borges: "Argumentum Ornithologicum" habla de una visión de un segundo, durante el cual el narrador ve una bandada de pájaros. De allí, a través de una meditación lógica en un par de líneas se llega a la conclusión de que Dios existe. Parece que la característica más llamativa del texto de Borges, respecto del género, es la extensión, por lo que el texto se clasifica dentro del subgrupo de minicuentos: supongo que la inclusión en este grupo no surgió en comparación con otros minicuentos, muchas veces de una sola línea, sino en comparación con los demás textos borgianos, notablemente más largos. Ahora: si consideramos los temas de Borges tratados en “Argumentum Ornithologicum”, vemos que la meditación sobre Dios, así como la mención de unos pájaros imaginarios son temas marcadamente poéticos; si no atendemos a la forma sino al tema, el texto bien podría incluirse en el corpus poético del autor. El razonamiento lógico a su vez evoca el método del ensayo. Extensión, tema y estructura fragmentan el texto y lo hacen partícipe de tres géneros diferentes, un poco como ocurre con la obra por todos conocida de Joseph Kosuth, One and Three Chairs (1965). La silla, su fotografía y su definición hablan de un problema teórico del arte, y su forzada inclusión en uno de los géneros de los que forma parte sería un grave error. Lo mismo ocurre con Borges y seguramente ocurre algo muy parecido con los "artículos" denominados anticuento de Juan José Millás.

En los tres ejemplos anteriores pasamos revista a una novela, unos poemas y algún que otro artículo; en dos casos aparecieron elementos intertextuales, en el tercero observamos más bien reflexión en vez de narración. No se vislumbra ningún denominador común. Y sin embargo parece evidente que el anticuento niega, se define en la oposición. Pero ¿qué es lo

\footnotetext{
4 https://www.amazon.com/El-anticuento-cuento-diferente-Spanish/dp/1977841732, última consulta: $12 / 09 / 2019$.

5 https://www.amazon.es/Anticuentos-Literaria-Editorial-Mill\%C3\%A1s-Garc\%C3\%ADa/dp/8484280985, última consulta: 12/09/2019.
} 
que niega exactamente? Negarlo todo a la vez es imposible, lo vimos en un sinfín de casos de las artes de vanguardia. Si sin embargo se lograra, el resultado sería la silla anteriormente mencionada, en la que ya no se reconoce la rebelión, sino más bien el uso práctico, ajeno a toda finalidad estética. Es tan anti que queda totalmente fuera, no guarda ninguna relación que apuntara a ese otro objeto estético al que se opone. Podríamos negar entonces un único elemento constitutivo a la vez: la cronología, el espacio, los personajes... Sin embargo cuando el caso se da, llegamos a la paradoja de Stevick, los textos en cuestión todavía se incluyen en las antologías del cuento experimental. Si evocamos el cuento de Ribeyro y el de Ferré, vemos que el primero aparece en una antología tradicional, con toda seguridad como ejemplo del límite. Con su inclusión el editor afirma que "esto todavía es un cuento" o "hasta aquí llegan los límites del género". A su vez el texto de Ferré es ejemplo de la experimentación. Aunque el autor niegue, el editor nos sugiere que todavía permanece dentro de los marcos. Que a pesar de su negación comparte aquellas características que hacen que un texto sea un cuento.

A base de los pocos ejemplos observados parece que textos con características análogas pueden ser igualmente cuentos o cuentos experimentales, a la vez que el anticuento no contiene ningún elemento específico que lo distinga claramente del resto de los géneros. Si admitimos la necesidad de alguna relación entre cuento y anticuento para que la oposición sea reconocible, la definición empezaría así: "el anticuento es un cuento que...", frase que en sí encierra una paradoja, una explosión. Es como el título de la antología de Stevick: "AntiStory" se presenta como una antología del cuento experimental, afirmación y negación se hallan en el mismo bando. Se da entonces el caso de que existe un corpus que contiene elementos impresionantemente parecidos (si no idénticos) a aquellos que quedan fuera de él. La misma definición ("el anticuento es un cuento que...”) de por sí destruye la del cuento: si el anticuento no se distingue esencialmente de ese otro al que se opone, y sin embargo se afirma como su polo opuesto, con este gesto crea una paradoja lógica que, a manera de un agujero negro, hunde todo lo que entra en su interior. Y por otra parte, si dentro del género del cuento hallamos una enorme diversidad (para no ir más lejos, el cuento experimental, toda la riqueza de la experimentación se comprendería dentro del conjunto de los cuentos): su oposición entonces tendría que ser un género que logre oponerse a todos los colores, a todas las formas y experimentos. Si esto fuese posible, el cuento como género se autodestruyera nada más con la mera mención de la posibilidad de que exista el anticuento.

David Antin propone otra posibilidad a la hora de hablar sobre un género. Observa que los participantes de aquellas disputas que pretenden fijar los límites de ciertos géneros nunca argumentan partiendo de definiciones: "Probablemente es así porque muy poca gente versada en el arte puede confiar en someras definiciones en un ámbito donde haya experimentado tanta ansiedad y esfuerzo, tanto placer y convicción; además este enfoque parece simplemente erróneo" (en línea). ${ }^{6}$

Como alternativa ofrece su propio ejemplo, el de la revista mencionada al principio de este artículo, titulada some/thing. Explica que la revista en cuestión publicaba toda una diversidad de textos definidos como poesía, interpretando el género como escenario de

\footnotetext{
6 "Probably this is so because very few people educated in art feel confident in sweeping definitions on a terrain in which they have experienced as much anxiety and effort as pleasure and conviction, but also because it simply seems the wrong way to go about it." La traducción es mía.
} 
diversas prácticas que se forma a través de los recuerdos de aquellos espectadores que lo "visitaban", así como a través del "espíritu del lugar". Para poder definir la noción del "espíritu del lugar" es preciso tener presente las representaciones vistas en el lugar en cuestión y aquellas que soñamos ver en el futuro en el mismo espacio. Antin dice que las obras de los creadores que publicaban en some/thing pertenecían a géneros bien diversos, y los editores contribuían en gran medida en ampliar las posibilidades a través de una diversidad de las maneras de la publicación, incitando a los lectores a considerar todos aquellos textos divergentes como poesía.

¿Para qué sirve entonces y hacia dónde lleva la anterior discusión acerca de la distinción de cuento, cuento experimental y anticuento? La respuesta de Antin, me parece, igualmente puede ser una solución a nuestras inquietudes narrativas:

Las tácticas pueden ser convincentes o no, pueden ser brillantes o triviales dependiendo si las conexiones son sustanciales o accidentales. Lo que este tipo de argumentos tienen en común es que nos llevan a comprender con más profundidad las tradiciones del género y del arte, llamándonos la atención en relaciones tan productivas que antes ni siquiera hubiéramos sospechado y contribuyen en que el género siga satisfaciendo nuestras necesidades. Parece que este tipo de juego mantiene en vivo los géneros. ${ }^{7}$ (en línea, la cursiva es mía)

\section{Bibliografía}

ANDERSON IMBERT, Enrique (2007): Teoría y técnica del cuento. Barcelona: Ariel. [1979]

ANTIN, David (1987): “The Stranger at the Door". Disponible en https://www.thing.net/ grist/ld/koppany/antin-e.htm (última consulta: 3/12/2019).

BURGOS, Fernando (ed.) (1997): El cuento hispanoamericano en el siglo XX. Madrid: Castalia.

DOMÍNGUEZ HIDALGO, Antonio (2012): Anticuentos. Ciudad de México: Umbral. [1971]

MILLÁS, Juan José (2001): Anticuentos. Barcelona: Alba.

RODRIGO, Óscar (2017): Anticuento. CreateSpace Independent Publishing Platform, ISBN10: 1977841732.

SCHOLZ, László (ed.) (2001): Prosas de mareo. Antología del cuento experimental hispanoamericano. Budapest: Eötvös Kiadó.

(C) Mercédesz Kutasy

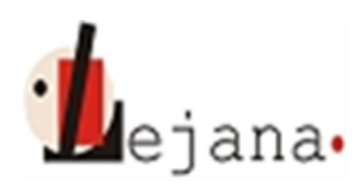

http://ojs.elte.hu/index.php/lejana

Universidad Eötvös Loránd, Departamento de Español, 1088 Budapest, Múzeum krt. 4/C

\footnotetext{
7 "These tactics can be convincing or unconvincing, brilliant or trivial, as the connections are fundamental or inconsequential. What most convincing arguments of this type have in common is some way of deeping sense of the tradition of the genre and of art by articulating some hitherto unknown and consequently unforeseen productive line of play that will allow the genre to continue to satisfy our needs. It appears to be this kind of game that keeps a genre alive". La cursiva es mía.
} 